\title{
Benefits of Investigating the Thermal Component for Moisture Safety in Ventilated Attics
}

\author{
Johan Claesson ${ }^{1, *}$ and Petter Wallentén ${ }^{1}$ \\ ${ }^{1}$ Lund University, DP Building Phyics, Box 118, 22100 Lund, Sweden
}

\begin{abstract}
Cold ventilated attics often have mould problems in Sweden. This is valid both for old and sometimes newly built attics. Increased insulation on the attic floor is assumed to increase the problem. To investigate this, numerical 1D models like WUFI or WUFI+ are typically used. These models give results but the physical processes are not so transparent for the user due to the complex numerical techniques involved and takes a long time to simulate. The problem is mainly related to the temperature in the attic, the ventilation rate and possible of leaks from the living space. All exposed surfaces in the attic will buffer moisture variations. But if this buffering is neglected and the leakage is treated as a constant the moisture content in the attic is only dependent on the ventilation with outside air and the assumed leakage. This would make a pure thermal investigation meaningful. An analytical model for the thermal problem was developed that took into account radiation between the interior surfaces and the different boundary conditions at the outside and inside surfaces. Using this model a parameter study of exterior roofing insulation was done using a moisture transport model that only took into account convection exchange. The results were compared with WUFI Pro and WUFI+ simulations which included the moisture exchange between air and internal surfaces. The comparison showed that the pure thermal model gave, as expected, larger variations in relative humidity, but that the results were qualitatively very similar. This indicates that analytical solutions of thermal problems can be used as a base in qualitative investigations of certain combined heat and moisture problems.
\end{abstract}

\section{Introduction}

The points of interest for mould problems in a cold attic are e.g. the attic air and the interior attic roof surface. There are many possible ways to implement a thermal model of an attic. Using a one-dimensional model where the ventilated air space is modelled as a space between two parallel plates is especially common when the model includes moisture transport, [6]. Since even the thermal problem is quite complex given that the attic has many layers the methods used to solve the problem are typically numerical. This means that the results are based on numerical solutions of the discrete transport equations for heat and moisture in the space and time dimension. Equation (1) shows the standard heat transfer equation and (2) a discretization using the explicit finite difference method with $\Delta x$ as the discretization step in space:

$$
x: x_{1}, x_{2} \cdots x_{N x} \quad x_{i+1}-x_{i}=\Delta x
$$

and $\Delta t$ the step in time:

$$
\begin{gathered}
t: t^{1}, t^{2} \cdots t^{N t} \quad t^{i+1}-t^{i}=\Delta t . \\
c \rho \frac{\partial T}{\partial t}=\frac{\partial}{\partial x}\left(\lambda \frac{\partial T}{\partial x}\right)
\end{gathered}
$$

$$
c \rho \frac{T_{i}^{n+1}-T_{i}^{n}}{\Delta t}=\lambda \frac{T_{i+2}^{n}-2 T_{i}^{n}+T_{i-2}^{n}}{\Delta x^{2}}
$$

Here is:

$\begin{array}{lll}T=T(x, t)\left[{ }^{\circ} \mathrm{C}\right] & \text { temperature } \\ t & {[\mathrm{~s}]} & \text { time } \\ x & {[\mathrm{~m}]} & \text { position, } \\ \lambda & {[\mathrm{W} / \mathrm{m} \mathrm{K}]} & \text { thermal conductivity, } \\ \mathrm{c} & {[\mathrm{J} / \mathrm{kg} \mathrm{K}]} & \text { heat capacity, } \\ \rho & {[\mathrm{kg} / \mathrm{m} 3]} & \text { density }\end{array}$

Results based on equation (2) will be increasingly more accurate with decreasing step in space and time and are dependent on the choice of discretization method, e.g. implicit or explicit methods. If there are areas in the material or certain time intervals that that need a smaller step this must be known in advance. Results based on equation (1), so called analytical solutions, are exact, meaning that that there are no other parameters that affects the results.

The paper presented here deals with an analytical solution of the transient heat transfer problem of an attic based on a thermal network. With this solution it is possible to do fast parameter studies with hourly climate data. This includes the effect of shortwave absorption coefficients, insulation thickness, ventilation rate etc.

\footnotetext{
* Corresponding author: petter.wallenten $@$ byggtek.lth.se
} 


\section{Thermal modelling of an attic}

In Sweden there is always a vapour barrier between the living space and the attic. This means that the moisture content in the attic is the same as in the outside air. This would make a pure thermal investigation meaningful. Since we could not find any published analytical solution to the problem we decided to investigate this. Our model consists of an attic floor, roof and a ventilated space in between. The heat transfer in the floor and roof is one dimensional but the radiation exchange between the surfaces takes into account the two dimensional view factors between the floor and roof. The floor consists of insulation. The composite roof consists of wood, insulation, a non-ventilated air gap and roof tiles. This is described in Figures 1 and 2. The heat transfer process is a linear one, which therefore includes linearization of the convective and radiative heat transfer at the surfaces. optional and left as a decision for the author. Where the author wishes to divide the paper into sections the formatting shown in Table 2 should be used.

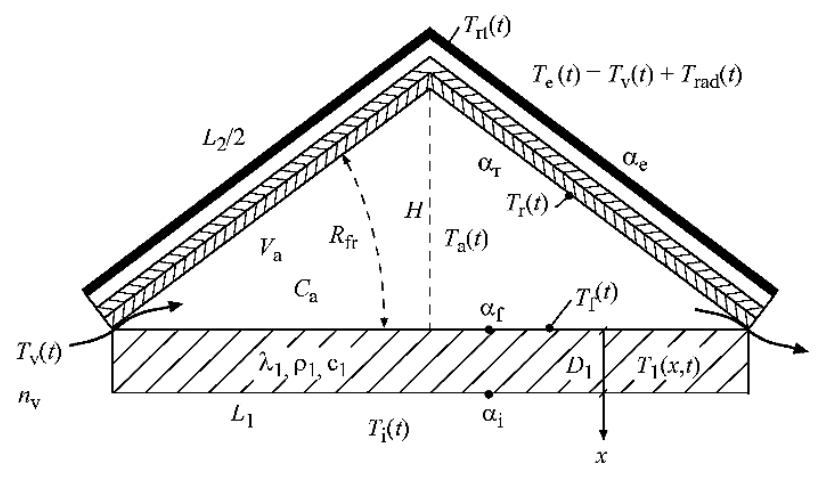

Fig. 1. Notations for an attic with radiation heat exchange between floor and roof

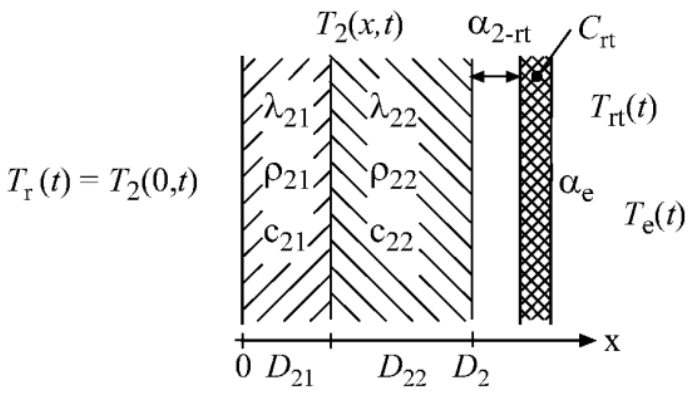

Fig. 2. Details of the composite roof involving two material layers and an air gap below roofing tiles

The following subscripts will be used: $a=$ attic air, $f=$ attic floor surface, $r=$ attic roof surface, $v=$ ventilation air temperature, $e=$ exterior air temperature, $i=$ interior air temperature, $r a d=$ effective outdoor air temperature due to radiation, 1 = insulation slab in attic floor, $21=$ wood slab in attic roof, 22 = insulation slab in attic roof, $r t=$ roofing tiles.
The model can be represented as thermal network which is described in Figure 3. It is easy to see the possible paths of the heat flow.

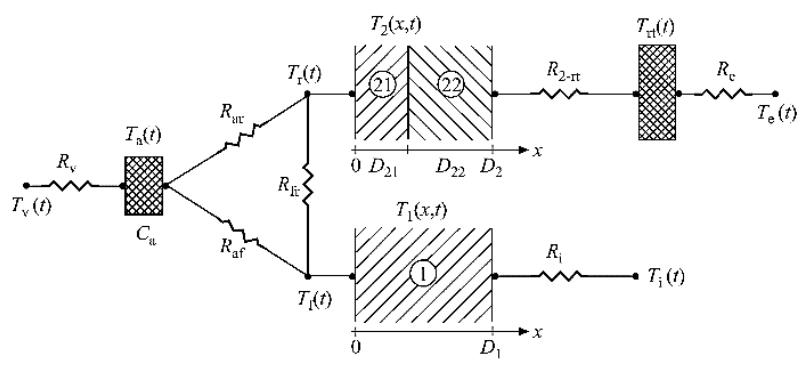

Fig. 3. Network to represent the thermal interactions and equations in the attic components

The thermal process is governed by the three prescribed boundary functions for ventilation, exterior and interior temperature:

$$
T_{\mathrm{v}}(t), \quad T_{\mathrm{e}}(t), \quad T_{\mathrm{i}}(t)
$$

These particular boundary conditions are chosen because they are simple but allow for a realistic treatment of the climate. Obvious simplifications are: constant ventilation rate, constant convective and radiative heat transfers coefficients, well mixed air in the attic space and that the influence of short and longwave radiation on the exterior surface can be simplified as an equivalent exterior temperature. The task is to calculate temperatures as functions of time at the four nodes $(\mathrm{a}, \mathrm{f}, \mathrm{r}, \mathrm{rt}$ ) and the temperature field through floor and roof:

$$
\begin{aligned}
& T_{a}(t), T_{f}(t), T_{r}(t), T_{r t}(t), T_{1}(x, t) \\
0 \leq & x \leq D_{1}, T_{2}(x, t) \quad 0 \leq x \leq D_{2}
\end{aligned}
$$

The temperature of the attic air, $T_{a}(t)$, and the interior surface of the attic roof, $T_{r}(t)=T_{2}(0, t)$, are of particular interest for studies of moisture problems. There is a heat balance equation at each node $(\mathrm{a}, \mathrm{f}, \mathrm{r}, \mathrm{rt})$. The equation for the air node, $T_{a}(t)$, is:

$$
C_{\mathrm{a}} \times \frac{d T_{\mathrm{a}}}{d t}=\frac{T_{\mathrm{v}}(t)-T_{\mathrm{a}}(t)}{R_{\mathrm{v}}}+\frac{T_{\mathrm{r}}(t)-T_{\mathrm{a}}(t)}{R_{\mathrm{ar}}}+\frac{T_{\mathrm{f}}(t)-T_{\mathrm{a}}(t)}{R_{\mathrm{af}}} .
$$


The equation for the node at the surface of the attic roof, $T_{r}(t)$, is, Figure 3 :

$$
\begin{gathered}
\frac{T_{a}(t)-T_{r}(t)}{R_{a r}}+\frac{T_{f}(t)-T_{r}(t)}{R_{f r}}=q_{2}(0, t)= \\
=L_{2} \cdot\left(-\lambda_{21}\right) \frac{\partial T_{2}}{\partial x} \quad x=0
\end{gathered}
$$

The heat equation for the temperature $T_{1}(x, t)$ in the insulation slab of the attic floor reads:

$$
\frac{1}{a_{1}} \cdot \frac{\partial T_{1}}{\partial t}=\frac{\partial^{2} T_{1}}{\partial x^{2}}, \quad 0 \leq x \leq D_{1}, a_{1}=\frac{\lambda_{1}}{\rho_{1} c_{1}}
$$

The heat equation for the temperature $T_{2}(x, t)$ of the composite roof is similar. The basic input data with values for the reference case are chosen as a relatively small attic:

$$
\begin{aligned}
& L_{1}=8 \mathrm{~m}, H=3 \mathrm{~m}, n_{v}=2 \mathrm{ach}, \rho_{\mathrm{a}}=1.29 \mathrm{~kg} / \mathrm{m} 3, \\
& c_{a}=1000 \mathrm{~J} / \mathrm{kg}, e=0.9, T_{a v}=283 \mathrm{~K}, a_{f}=4 \mathrm{~W} / \mathrm{m}^{2} \mathrm{~K}, \\
& D_{1}=0.4 \mathrm{~m}, \lambda_{1}=0.04 \mathrm{~W} / \mathrm{mK}, \rho_{1}=20 \mathrm{~kg} / \mathrm{m}^{3}, \\
& c_{1}=800 \mathrm{~J} / \mathrm{kg}, a_{i}=8 \mathrm{~W} / \mathrm{m}^{2} \mathrm{~K}, a_{r}=4 \mathrm{~W} / \mathrm{m}^{2} \mathrm{~K}, \\
& D_{21}=0.02 \mathrm{~m}, L_{21}=0.14 \mathrm{~W} / \mathrm{mK}, \rho_{21}=500 \mathrm{~kg} / \mathrm{m}^{3}, \\
& c_{21}=1200 \mathrm{~J} / \mathrm{kg}, D_{22}=0.55 \mathrm{~m}, \lambda_{22}=0.04 \mathrm{~W} / \mathrm{mK}, \\
& \rho_{22}=50 \mathrm{~kg} / \mathrm{m}^{3}, c_{22}=800 \mathrm{~J} / \mathrm{kg}, a_{2 r t}=15 \mathrm{~W} / \mathrm{m}^{2} \mathrm{~K}, \\
& D_{r t}=0.015 \mathrm{~m}, \rho_{r t}=1500 \mathrm{~kg} / \mathrm{m}^{3}, c_{r t}=800 \mathrm{~J} / \mathrm{kg}, \\
& a_{e}=15 \mathrm{~W} / \mathrm{m}^{2} \mathrm{~K}
\end{aligned}
$$

\section{Step responses for the three basic cases}

The determination of the attic temperatures is based on the solutions for three basic cases, one for each boundary condition. In the first case associated with the ventilation boundary, the ventilation temperature experiences a unit temperature step from 0 to 1 at $t=0$. The exterior and interior boundary temperatures are zero for all times. The temperature at the start $t=0$ is zero in the whole attic. Three basic step-response problems (ventilation, exterior, interior) are defined by the following boundary conditions:

$$
\begin{gathered}
\text { Ventilation: } T_{v}(t)=H(t), T_{e}(t)=0, T_{i}(t)=0 \\
\text { Exterior: } T_{e}(t)=H(t), T_{v}(t)=0, T_{i}(t)=0 \\
\text { Interior: } T_{i}(t)=H(t), T_{e}(t)=0, T_{v}(t)=0 \\
\text { with: } H(t>0)=1, \quad H(t<0)=0
\end{gathered}
$$

\section{Step responses for ventilation temperature}

The solution for a step in the ventilation boundary temperature involves the following temperature components (using a bold face superscript $\mathrm{v}$ for the ventilation step response):

$$
U_{a}^{v}(t), U_{f}^{v}(t), U_{r}^{v}(t), U_{r t}^{v}(t), U_{1}^{v}(x, t) \text { for } 0 \leq x \leq D_{1} \text {, }
$$

$U_{2}^{v}(x, t)$ for $0 \leq x \leq D_{2}$

These functions describe the step response at all points in the thermal model. Some examples of solutions are given in Figure 4



Fig. 4. Temperature distribution through roof $(-0.07<x$ $<0)$ and floor $(0<x<0.4)$ for the ventilation step for $t=$ $0.1, \ldots, 10$ hours. The top curve shows the steady-state temperature.

\section{Solution techniques}

A methodology called dynamic thermal networks to solve thermal problems involving transient heat conduction have been presented by Claesson and co-workers in a number of papers. These networks represent the relations between boundary heat fluxes and boundary temperatures. The current heat fluxes are obtained by integrals (or sums) of preceding boundary temperatures multiplied by weighting functions, [1-2]. The theory is applied to composite walls in [3], to a building with walls, roof and foundation in $[4,5]$. Solution techniques involving Laplace transforms and Fourier series with determination of eigenvalues are used. This whole system with all its complex interactions may be represented by a thermal network for the Laplace transform and for the eigenvalues. From these similar networks the Laplace transform and the equation for eigenvalues are readily obtained. The final Laplace solution is obtained by an integral inversion. The full thermal model for the attic requires some four pages of formulas and relations in Mathcad. The computer time for about five digits accuracy is a few minutes. 


$$
\begin{aligned}
& U_{1}(x, t)=U_{1, \text { stat }}(x)-\sum_{n} a_{n} \cdot U_{1, n}(x) \cdot e^{-v_{n} t} \\
& U_{2}(x, t)=U_{2, \text { stat }}(x)-\sum_{n} a_{n} \cdot U_{2, n}(x) \cdot e^{-v_{n} t} \\
& U_{a}(t)=U_{a, \text { stat }}-\sum_{n} a_{n} \cdot U_{a, n} \cdot e^{-v_{n} t} \\
& U_{r t}(t)=U_{r t, \text { stat }}-\sum_{n} a_{n} \cdot U_{r t, n} \cdot e^{-v_{n} t}
\end{aligned}
$$

\subsection{General superposition formula}

Let $\mathbf{P}$ denote any considered point (node, point in floor or roof) for which the temperature is to be determined:

$$
\begin{aligned}
& \text { P: } a, f, r, r t, x=x_{1}\left(0 \leq x \leq D_{1}\right), \\
& x=x_{2}\left(0 \leq x \leq D_{2}\right)
\end{aligned}
$$

The temperature at $\mathbf{P}$ as function of time $t$ depends on the three boundary temperatures taken for preceding times up to time t. General superposition gives the following exact formula:

$$
\begin{gathered}
T_{\mathbf{P}}(t)=\int_{0}^{\infty}\left[W_{\mathbf{P}}^{v}(\tau) \cdot T_{v}(t-\tau)+W_{\mathbf{P}}^{e}(\tau) \cdot T_{e}(t-\tau)+\right. \\
\left.W_{\mathbf{P}}^{i}(\tau) \cdot T_{i}(t-\tau)\right] d \tau
\end{gathered}
$$

Here, the weighting functions are given by the time derivative of the three basic step-response solutions at the considered point $\mathbf{P}$ :

$$
\begin{gathered}
W_{\mathbf{P}}^{v}(\tau)=\frac{d}{d \tau}\left[U_{\mathbf{P}}^{v}(\tau)\right], \quad W_{\mathbf{P}}^{e}(\tau)=\frac{d}{d \tau}\left[U_{\mathbf{P}}^{e}(\tau)\right], \\
W_{\mathbf{P}}^{i}(\tau)=\frac{d}{d \tau}\left[U_{\mathbf{P}}^{i}(\tau)\right],
\end{gathered}
$$

The weighting factors are positive (or zero), since the Ufunctions increase monotonously with time, and the derivatives tend to zero (exponentially) for large times. Equation (12) thus expresses the full analytical solution of the problem. No discretization or numerical limitations are used at this point.

\subsection{Discretization}

If the boundary conditions change stepwise, which is typical for measured data, they can be expressed as constant for each time interval $n$ :

$$
\begin{aligned}
& T_{v}(t)=T_{v, n}, \quad T_{e}(t)=T_{e, n}, \quad T_{i}(t)=T_{i, n} \\
& \text { for }(n-1) h<t<n \cdot h
\end{aligned}
$$

Here, $h$ is the time step, which often is typically $h=1$ hour. Formula (12) gives:

$$
\begin{gathered}
T_{\mathbf{P}}(n t)=T_{\mathbf{P}, n}=\sum_{v=1}^{\infty} \int_{v h-h}^{v h}\left[W_{\mathbf{P}}^{v}(\tau) \cdot T_{v, n-v}+W_{\mathbf{P}}^{e}(\tau)\right. \\
\left.T_{e, n-v}+W_{\mathbf{P}}^{i}(\tau) \cdot T_{i, n-v}\right] d \tau
\end{gathered}
$$

The sum of all weighting factors becomes equal to one. Equation (15) may therefore be written in the following way:

$$
\begin{gathered}
\sum_{v=1}^{\infty}\left[W_{\mathbf{P}, v}^{v} \cdot T_{v, n-v}-T_{\mathbf{P}, n}+W_{\mathbf{P}, v}^{e} \cdot T_{e, n-v}-T_{\mathbf{P}, n}+\right. \\
\left.W_{\mathbf{P}, v}^{i} \cdot T_{i, n-v}-T_{\mathbf{P}, n}\right]=0
\end{gathered}
$$

This relation may be represented graphically as a kind of dynamic thermal network, Figure 5.

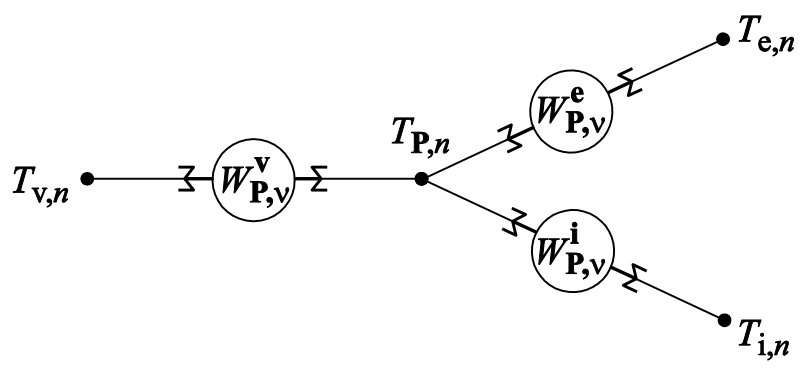

Fig. 5. Dynamic thermal network to represent the weighting formulas (16).

Data from the reference case (8) gives that sum above may be truncated after 15 time steps of one hour each.

\section{Parameter study. An example}

With the model and solution described above it is easy to investigate the importance of the parameters in the model for any given boundary conditions. As an example has the importance of the exterior insulation thickness been studied using a climate in Lund for one year (1990). When investigating moisture related issues it is of interest to calculate the risk of high relative humidity on the attic roof surface which is directly dependent on the difference between the outdoor (ventilation) temperature and the roof surface Diff $=T_{r}-T_{v}$. The risk for mould problems increases with Diff. Figure 6 shows Diff for a year in Lund 1990 presented in cumulative histogram form. The $\mathrm{x}$ axis is Diff and the y axis is the number of hours the value is below Diff. The calculations show that $1 \mathrm{~cm}$ of exterior insulation does not reduce the number of hours with cooling of the ventilated air. There are about 4500 hours 
with cooling. With $5 \mathrm{~cm}$ insulation, as in Figure 7 however, does the number of hours with cooling of ventilates air go down to about 2500 hours.



Fig. 6. Cumulative histogram of Diff from hourly simulation of year 1990 in Lund, Sweden. Exterior insulation thickness $0 \mathrm{~cm}$.

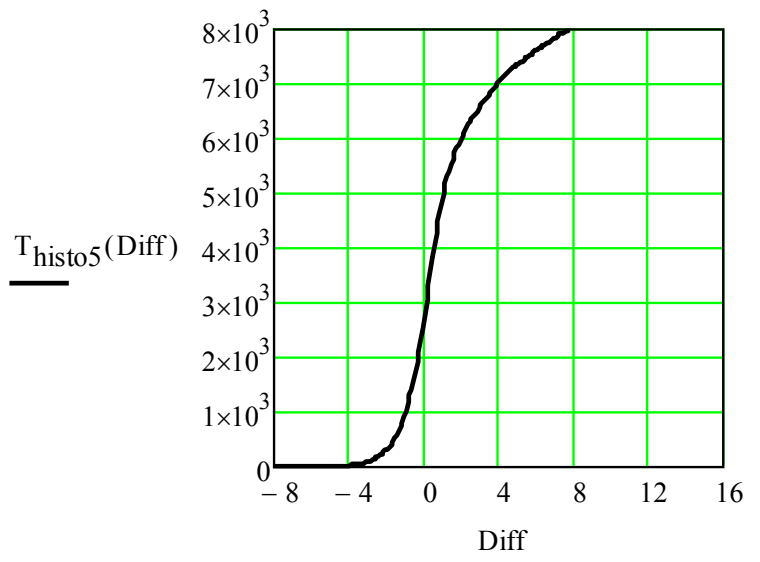

Fig. 7. Cumulative histogram of Diff from hourly simulation of year 1990 in Lund, Sweden. Exterior insulation thickness $5 \mathrm{~cm}$.

\section{Discussion and comparison with other models}

The simplification that there is no moisture buffeting in the attic ceiling is of course affecting the results. To investigate this, simulations with three heat and moisture transfer programs were done with the same construction as above:

(1) 1D WUFI Pro [7] simulation with moisture transport in all materials (subscript_1D).

(2) WUFI+ [8] model $(12 \mathrm{~m} \cdot 8 \mathrm{~m} \cdot 3 \mathrm{~m})$ without moisture transport in the attic ceiling (subscript + ), see figure 8 .

(3) WUFI+ model $(12 \mathrm{~m} \cdot 8 \mathrm{~m} \cdot 3 \mathrm{~m})$ with moisture transport in all layers (subscript $\_{ }^{+m}$ ).

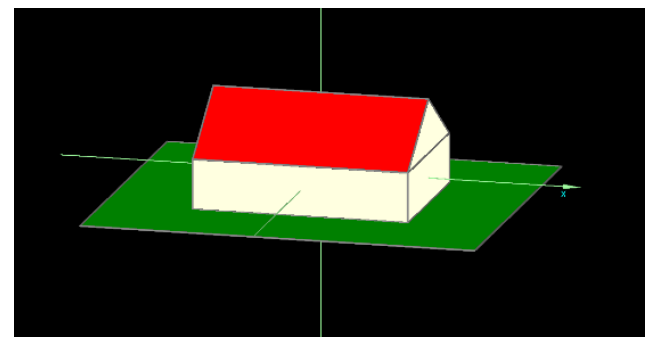

Fig. 8. WUFI+ model which includes. (The attic is the simulated zone)

Figures 9 and 10 show the temperature and relative humidity in the attic for a period during the autumn. All the WUFI programs use the implicit finite difference method for the solution of the transport equations, typically using the time step of one hour. The comparison showed that the pure thermal model gave, as expected, larger variations in relative humidity, but that the results were qualitatively very similar. The often used 1D WUFI Pro model gave surprisingly low relative humidity (and higher temperature). The results shown in figure 9 indicates that the analytical solution ( $\mathrm{T}_{-}$a) that takes into account geometry but not moisture transport, and the 1D WUFI Pro model (T_1D) which takes into account moisture transport but not the geometry are both extremes. That is not to say that the WUFI+ model is the "correct" solution, since it is a numerical solution, but in theory this model is the most complete one.

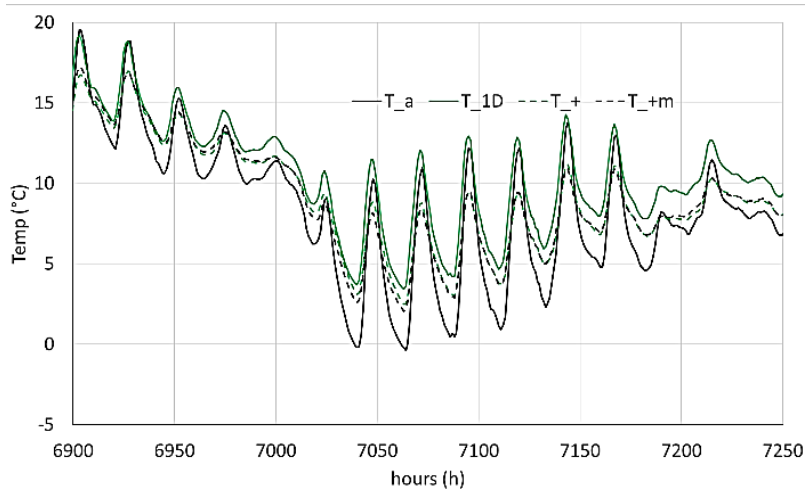

Fig. 9. Temperature in the attic calculated with the analytical solution (T_a), 1D WUFI Pro (T_1D), WUFI+ without moisture buffeting $\left(\mathrm{T}_{-}+\right)$and $\overline{\mathrm{WUFI}}+$ with moisture transport $\left(\mathrm{T}_{-}+\mathrm{m}\right)$ 


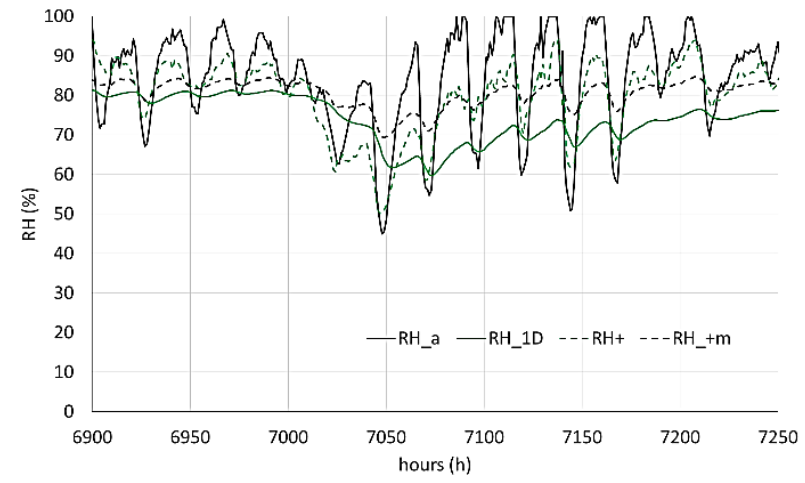

Fig. 10. Relative humidity the attic calculated with the analytical solution (T_a), 1D WUFI Pro (T_1D), WUFI+ without moisture buffeting $\left(\mathrm{T}_{-}+\right)$and $\overline{\text { WUFI}}+$ with moisture transport $\left(\mathrm{T}_{-}+\mathrm{m}\right)$.

\section{Conclusions}

The paper presents an analytical solution method based on dynamic thermal networks for the heat transfer problem in an attic with a pitched roof. Although there exists a number of numerical approaches to solve this problem (Energy + , IDA-ICE etc), no analytical solution has as far as the authors know been presented before. The solution shows the physical behavior of the different components in a very clear way. It is also easy to investigate the effect of the included parameters. Comparisons with other models show that the thermal problem is the dominant problem for a cold attic.

This project is part of WoodBuild, a research programme within the Sectoral R\&DProgramme 2006-2012 for the Swedish forestbased industry.

\section{References}

1. J. Claesson J Dynamic Thermal Networks. Outlines of a General Theory. 6th Symposium on Building Physics in the Nordic Countries, June 2002, Trondheim, Norway. (2002)

2. J. Claesson. Dynamic Thermal Networks. A Methodology to Account for Time-dependent Heat Conduction. Sec. Int. Building Physics Conference, Sept. Leuven, Belgium. (2003)

3. E.-L.Wentzel,J. Claesson. Heat Loss Dynamics of Walls. Analysis and Optimizing Based on the Theory of Dynamic Thermal Networks. Sec. Int. Building Physics Conference, Sept. 2003, Leuven, Belgium. (2003)

4. E.-L.Wentzel, J. Claesson J. Heat Loss to Building Foundations. An Analyses Based on a Theory of Dynamic Thermal Networks. Performance of Exterior of Whole Buildings. Ninth Int. Conf., Florida, Dec. (2004).

5. E.-L. Wentzel. Thermal Modelling of Walls, Foundations and Whole Buildings Using
Dynamic Thermal Networks. Thesis, Dept. of Civil and Env. Eng., Div. of Building Technology, Chalmers Univ. of Technology, Gothenburg, Sweden. (2005)

6. M. N.Vahid, A. Sasic Kalagasidis, E. Kjellström Energy and Buildings Assessment of hygrothermal performance and mould growth risk in ventilated attics in respect to possible climate changes in Sweden, Building and Environment 55 96-109, (2012)

7. A. Holm and H. M. Künzel, Non-Isothermal Moisture Transfer in Porous Building Materials, Materialsweek (2000)

8. F. Antretter, F. Sauer, T. Schöpfer and A. Holm, Validation of a hygrothermal whole building simulation software, Proceedings of Building Simulation 2011: 12th Conference of International Building Performance Simulation Association, Sydney, 14-16 November (2011) 